\title{
Symplectic Applicability of Lagrangian Surfaces ${ }^{\star}$
}

\author{
Emilio MUSSO ${ }^{\dagger}$ and Lorenzo NICOLODI ${ }^{\ddagger}$
}

† Dipartimento di Matematica, Politecnico di Torino, Corso Duca degli Abruzzi 24, I-10129 Torino, Italy

E-mail: emilio.musso@polito.it

¥ Dipartimento di Matematica, Università degli Studi di Parma, Viale G.P. Usberti 53/A, I-43100 Parma, Italy

E-mail: lorenzo.nicolodi@unipr.it

Received February 25, 2009, in final form June 15, 2009; Published online June 30, 2009 doi:10.3842/SIGMA.2009.067

\begin{abstract}
We develop an approach to affine symplectic invariant geometry of Lagrangian surfaces by the method of moving frames. The fundamental invariants of elliptic Lagrangian immersions in affine symplectic four-space are derived together with their integrability equations. The invariant setup is applied to discuss the question of symplectic applicability for elliptic Lagrangian immersions. Explicit examples are considered.
\end{abstract}

Key words: Lagrangian surfaces; affine symplectic geometry; moving frames; differential invariants; applicability

2000 Mathematics Subject Classification: 53A07; 53B99; 53D12; 53A15

\section{Introduction}

While the subject of metric invariants of Lagrangian submanifolds has received much attention in the literature, the subject of affine symplectic invariants of Lagrangian submanifolds has been less studied (cf. [21] for recent work in this direction). Early contributions to symplectic invariant geometry of submanifolds go back to Chern and Wang [10], who studied submanifolds in projective $(2 n+1)$-space under the linear symplectic group. Some recent works related to the study of affine symplectic invariants, mainly for the case of curves and hypersurfaces in Euclidean space, include $[11,19,1]$. The aim of this paper is to develop an approach to affine symplectic invariant geometry of Lagrangian surfaces in standard affine symplectic four-space, using the method of moving frames. The invariant setup is then applied to discuss the problem of symplectic applicability for Lagrangian immersions.

Let $M$ be a 2-dimensional manifold and let $f: M \rightarrow \mathbb{R}^{4}$ be a Lagrangian immersion into standard affine symplectic four-space $\left(\mathbb{R}^{4}, \Omega\right)$. The basic observation is that the natural Gauss map of $f$, whose value at a point is determined by the 1 -jet of $f$ at that point, takes values in the oriented Lagrangian Grassmannian of $\left(\mathbb{R}^{4}, \Omega\right)$, the manifold $\Lambda_{2}^{+}$of oriented Lagrangian 2 -subspaces in $\mathbb{R}^{4}$. The oriented Lagrangian Grassmannian admits an alternative description as the conformal compactification of Minkowski 3-space $\mathbb{R}^{2,1}$, the projectivization of the positive nullcone of $\mathbb{R}^{3,2}$. The local isomorphisms between the group $\operatorname{Sp}(4, \mathbb{R})$ of linear symplectomorphisms and the conformal Lorentz group $\mathrm{O}(3,2)$ establishes a close relationship between the symplectic geometry of a Lagrangian surface in $\mathbb{R}^{4}$ and the conformal Lorentzian geometry of its Gauss map. Accordingly, a Lagrangian immersion is of general type (resp., special type) if its Gauss map is an immersion (resp., has constant rank one). Moreover, a Lagrangian immersion

\footnotetext{
*This paper is a contribution to the Special Issue "Élie Cartan and Differential Geometry". The full collection is available at http://www.emis.de/journals/SIGMA/Cartan.html
} 
of general type is elliptic (resp., hyperbolic, parabolic) if its Gauss map is a spacelike (resp., timelike, lightlike) immersion into $\Lambda_{2}^{+}$.

In this work we study elliptic Lagrangian immersions in $\left(\mathbb{R}^{4}, \Omega\right)$ under the group $\mathbb{R}^{4} \rtimes \operatorname{Sp}(4, \mathbb{R})$ of affine symplectic transformations. In Section 2, we collect the background material on affine symplectic frames and their structure equations, and briefly describe the conformal geometry of the Lagrangian Grassmannian $\Lambda_{2}^{+}$.

In Section 3, we develop the moving frame method for elliptic Lagrangian immersions in affine symplectic geometry. If $f: M \rightarrow \mathbb{R}^{4}$ is an elliptic Lagrangian immersion, we consider on $M$ the complex structure defined by the conformal structure induced by the Gauss map of $f$ and a choice of orientation on $M$. After the third frame reduction, we make a successive reduction with respect to a fixed local complex coordinate in the Riemann surface $M$, which yields a unique adapted frame field along $f$. From this we derive the three local affine symplectic invariants for $f$, namely the complex-valued smooth functions $t, h, p: M \rightarrow \mathbb{C}$, and establish their integrability equations, which give the existence and uniqueness theorem for elliptic Lagrangian immersion in affine symplectic geometry. At this stage, a natural question to ask is to what extent the invariants are actually needed to determine the elliptic Lagrangian surface up to symmetry (cf. Bonnet's problem in Euclidean geometry). We will look at this problem from the classical point of view of applicable surfaces, a concept generalized by É. Cartan to $G$-deformations of submanifolds of any homogeneous space $G / G_{0}$ (cf. $[12,8,9,14,17])$.

In Section 4, we apply the invariant setup to discuss rigidity and applicability of elliptic Lagrangian immersions. Already after the second reduction, we associate with any elliptic Lagrangian immersion $f$ a cubic differential form $\mathcal{F}$, the Fubini cubic form. Proposition 2 shows that the position of a generic elliptic Lagrangian immersion is completely determined by $\mathcal{F}$, up to affine symplectic transformation. The elliptic Lagrangian immersions which are not determined by the Fubini cubic form alone are called applicable. Theorem 2 relates applicability to the complex structure of $M$, using the normalized Hopf differential $\mathcal{H}$, a quadratic differential form naturally associated to any elliptic Lagrangian immersion with respect to third order frames. More precisely, it proves that an elliptic Lagrangian immersion is applicable if and only if there exists on $M$ a non-zero holomorphic quadratic differential $\mathcal{Q}$ such that $\mathcal{H}$ and $\mathcal{Q}$ are linearly dependent over the reals at each point of $M$. An interesting consequence of this result is that applicable elliptic Lagrangian immersions admit an associated family of noncongruent elliptic Lagrangian immersions parametrized by $\mathbb{R}$, which in turn suggests their integrable character. This is not surprising when one considers that the Gauss map of an applicable elliptic Lagrangian immersion is an isothermic spacelike immersion of $\Lambda_{2}^{+}$. Indeed, it is well known that a spacelike isothermic immersion in 3-dimensional conformal space comes in an associated family of (second order) deformations which coincide with the classical $T$-transforms of Bianchi and Calapso (cf. [22, 16, 24]). The equations governing elliptic Lagrangian immersions are also derived. We then consider the class of elliptic Lagrangian surfaces with totally umbilic Gauss map. They are defined by the vanishing of the normalized Hopf differential $\mathcal{H}$. Proposition 3 shows that, up to a biholomorphism of $M$, two noncongruent, totally umbilic, elliptic Lagrangian immersions are applicable. Finally, Proposition 4 proves that a complex curve in $\mathbb{C}^{2}$ without flex points is a totally umbilic elliptic Lagrangian immersion and that, conversely, any totally umbilic elliptic Lagrangian immersion is congruent to a complex curve in $\mathbb{C}^{2}$ without flex points.

Section 5 is devoted to the analysis of explicit examples of applicable non-totally umbilic elliptic Lagrangian immersions. 


\section{Preliminaries}

\subsection{Affine symplectic frames and structure equations}

Let $\mathbb{R}_{\Omega}^{4}$ denote $\mathbb{R}^{4}$ with the standard symplectic form

$$
\Omega(X, Y)={ }^{t} X J Y, \quad X, Y \in \mathbb{R}^{4},
$$

where

$$
J=\left(J_{i j}\right)=\left(\begin{array}{cc}
0 & I_{2} \\
-I_{2} & 0
\end{array}\right)
$$

$I_{2}$ being the $2 \times 2$ identity matrix. The linear symplectic group is

$$
\operatorname{Sp}(4, \mathbb{R})=\left\{\mathbf{X} \in \mathrm{GL}(4, \mathbb{R}):{ }^{t} \mathbf{X} J \mathbf{X}=J\right\} .
$$

An element $\mathbf{X} \in \operatorname{Sp}(4, \mathbb{R})$ is of the form

$$
\mathbf{X}=\left(\begin{array}{cc}
A & B \\
C & D
\end{array}\right)
$$

where $A, B, C$ and $D$ are $2 \times 2$ matrices such that

$$
{ }^{t} A C-{ }^{t} C A=0, \quad{ }^{t} B D-{ }^{t} D B=0, \quad{ }^{t} A D-{ }^{t} C B=I_{2} .
$$

The Lie algebra of $\operatorname{Sp}(4, \mathbb{R})$ is

$$
\mathfrak{s p}(4, \mathbb{R})=\left\{\mathbf{x}(a, b, c)=\left(\begin{array}{cc}
a & b \\
c & -{ }^{t} a
\end{array}\right): a \in \mathfrak{g l}(2, \mathbb{R}), c, b \in \mathrm{S}(2, \mathbb{R})\right\}
$$

where $S(2, \mathbb{R})$ is the vector space of $2 \times 2$ symmetric matrices endowed with the inner product of signature $(2,1)$ defined by

$$
(b, b)=-\operatorname{det} b, \quad b \in \mathrm{S}(2, \mathbb{R}) .
$$

The affine symplectic group $\mathbb{R}^{4} \rtimes \mathrm{Sp}(4, \mathbb{R})$ is represented in $\operatorname{GL}(5, \mathbb{R})$ by

$$
\mathcal{S}(4, \mathbb{R})=\left\{\mathbf{S}(P, \mathbf{X})=\left(\begin{array}{cc}
1 & 0 \\
P & \mathbf{X}
\end{array}\right): P \in \mathbb{R}^{4}, \mathbf{X} \in \operatorname{Sp}(4, \mathbb{R})\right\}
$$

The Lie algebra of $\mathcal{S}(4, \mathbb{R})$ is given by

$$
\mathfrak{s}(4, \mathbb{R})=\left\{\mathrm{S}(p, \mathbf{x})=\left(\begin{array}{cc}
0 & 0 \\
p & \mathbf{x}
\end{array}\right): p \in \mathbb{R}^{4}, \mathbf{x} \in \mathfrak{s}(4, \mathbb{R})\right\} .
$$

If $\mathbf{S}(P, \mathbf{X}) \in \mathcal{S}(4, \mathbb{R})$, we let $X_{j}, j=1, \ldots, 4$, denote the column vectors of $\mathbf{X}$.

By an affine symplectic frame is meant a point $P \in \mathbb{R}^{4}$ and four vectors $X_{1}, \ldots, X_{4} \in \mathbb{R}^{4}$ such that $\mathbf{X}=\left(X_{1}, X_{2}, X_{3}, X_{4}\right) \in \operatorname{Sp}(4, \mathbb{R})$. Upon choice of a reference frame, the manifold of all affine symplectic frames of $\mathbb{R}_{\Omega}^{4}$ may be identified with the group $\mathcal{S}(4, \mathbb{R})$.

Consider the tautological projection maps

$$
\begin{aligned}
& \mathbf{P}: \mathcal{S}(4, \mathbb{R}) \ni \mathbf{S}(P, \mathbf{X}) \mapsto P \in \mathbb{R}^{4} \\
& \mathbf{X}_{j}: \mathcal{S}(4, \mathbb{R}) \ni \mathbf{S}(P, \mathbf{X}) \mapsto X_{j} \in \mathbb{R}^{4},
\end{aligned}
$$


for $j=1, \ldots, 4$. Then

$$
d \mathbf{P}=\tau^{j} \mathbf{X}_{j}, \quad d \mathbf{X}_{j}=\theta_{j}^{i} \mathbf{X}_{i},
$$

where the $\tau^{j}, \theta_{j}^{i}$ are the left-invariant Maurer-Cartan forms of $\mathcal{S}(4, \mathbb{R})$. Exterior differentiation of

$$
\Omega\left(\mathbf{X}_{i}, \mathbf{X}_{j}\right)=J_{i j}
$$

yields that $\theta$ is $\mathfrak{s p}(4, \mathbb{R})$-valued. We then write

$$
\theta=\left(\begin{array}{cc}
\alpha & \beta \\
\gamma & -{ }^{t} \alpha
\end{array}\right)
$$

where

$$
\alpha=\left(\begin{array}{ll}
\alpha_{1}^{1} & \alpha_{2}^{1} \\
\alpha_{1}^{2} & \alpha_{2}^{2}
\end{array}\right), \quad \beta=\left(\begin{array}{ll}
\beta_{1}^{1} & \beta_{2}^{1} \\
\beta_{2}^{1} & \beta_{2}^{2}
\end{array}\right), \quad \gamma=\left(\begin{array}{ll}
\gamma_{1}^{1} & \gamma_{2}^{1} \\
\gamma_{2}^{1} & \gamma_{2}^{2}
\end{array}\right) .
$$

Note that

$$
\left(\tau^{1}, \tau^{2}, \tau^{3}, \tau^{4}, \alpha_{1}^{1}, \alpha_{2}^{2}, \alpha_{2}^{1}, \alpha_{1}^{2}, \gamma_{1}^{1}, \gamma_{2}^{2}, \gamma_{2}^{1}, \beta_{1}^{1}, \beta_{2}^{2}, \beta_{2}^{1}\right)
$$

is a basis for the vector space of the left-invariant 1-forms of $\mathcal{S}(4, \mathbb{R})$. Moreover, for each $\mathbf{S}(0, \mathbf{Y}) \in \mathcal{S}(4, \mathbb{R})$, we have

$$
R_{\mathbf{S}(0, \mathbf{Y})}^{*}\left(\begin{array}{c}
\tau^{1} \\
\tau^{2} \\
\tau^{3} \\
\tau^{4}
\end{array}\right)=\mathbf{Y}^{-1}\left(\begin{array}{c}
\tau^{1} \\
\tau^{2} \\
\tau^{3} \\
\tau^{4}
\end{array}\right), \quad R_{\mathbf{S}(0, \mathbf{Y})}^{*}(\theta)=\mathbf{Y}^{-1} \theta \mathbf{Y}+\mathbf{Y}^{-1} d \mathbf{Y}
$$

Exterior differentiation of

$$
d \mathbf{P}=\tau^{j} \mathbf{X}_{j}, \quad d \mathbf{X}_{i}=\theta_{i}^{j} \mathbf{X}_{j}
$$

yields the structure equations of the affine symplectic group:

$$
\begin{aligned}
& \left\{\begin{array}{l}
d \tau^{1}=-\alpha_{1}^{1} \wedge \tau^{1}-\alpha_{2}^{1} \wedge \tau^{2}-\beta_{1}^{1} \wedge \tau^{3}-\beta_{2}^{1} \wedge \tau^{4}, \\
d \tau^{2}=-\alpha_{1}^{2} \wedge \tau^{1}-\alpha_{2}^{2} \wedge \tau^{2}-\beta_{2}^{1} \wedge \tau^{3}-\beta_{2}^{2} \wedge \tau^{4}, \\
d \tau^{3}=-\gamma_{1}^{1} \wedge \tau^{1}-\gamma_{2}^{1} \wedge \tau^{2}+\alpha_{1}^{1} \wedge \tau^{3}+\alpha_{1}^{2} \wedge \tau^{4}, \\
d \tau^{4}=-\gamma_{2}^{1} \wedge \tau^{1}-\gamma_{2}^{2} \wedge \tau^{2}+\alpha_{2}^{1} \wedge \tau^{3}+\alpha_{2}^{2} \wedge \tau^{4},
\end{array}\right. \\
& \left\{\begin{array}{l}
d \alpha_{1}^{1}=-\alpha_{2}^{1} \wedge \alpha_{1}^{2}-\beta_{1}^{1} \wedge \gamma_{1}^{1}-\beta_{2}^{1} \wedge \gamma_{2}^{1}, \\
d \alpha_{2}^{2}=-\alpha_{1}^{2} \wedge \alpha_{2}^{1}-\beta_{2}^{1} \wedge \gamma_{2}^{1}-\beta_{2}^{2} \wedge \gamma_{2}^{2}, \\
d \alpha_{1}^{2}=\left(\alpha_{1}^{1}-\alpha_{2}^{2}\right) \wedge \alpha_{1}^{2}-\beta_{2}^{1} \wedge \gamma_{1}^{1}-\beta_{2}^{2} \wedge \gamma_{2}^{1}, \\
d \alpha_{2}^{1}=\left(\alpha_{2}^{2}-\alpha_{1}^{1}\right) \wedge \alpha_{2}^{1}-\beta_{1}^{1} \wedge \gamma_{2}^{1}-\beta_{2}^{1} \wedge \gamma_{2}^{2},
\end{array}\right. \\
& \left\{\begin{array}{l}
d \gamma_{1}^{1}=2 \alpha_{1}^{1} \wedge \gamma_{1}^{1}+2 \alpha_{1}^{2} \wedge \gamma_{2}^{1}, \\
d \gamma_{2}^{2}=2 \alpha_{2}^{2} \wedge \gamma_{2}^{2}+2 \alpha_{2}^{1} \wedge \gamma_{2}^{1}, \\
d \gamma_{2}^{1}=\left(\alpha_{1}^{1}+\alpha_{2}^{2}\right) \wedge \gamma_{2}^{1}+\alpha_{1}^{2} \wedge \gamma_{2}^{2}+\alpha_{2}^{1} \wedge \gamma_{1}^{1},
\end{array}\right.
\end{aligned}
$$

and

$$
\left\{\begin{array}{l}
d \beta_{1}^{1}=-2 \alpha_{1}^{1} \wedge \beta_{1}^{1}-2 \alpha_{2}^{1} \wedge \beta_{2}^{1}, \\
d \beta_{2}^{2}=-2 \alpha_{2}^{2} \wedge \beta_{2}^{2}-2 \alpha_{1}^{2} \wedge \beta_{2}^{1}, \\
d \beta_{2}^{1}=-\left(\alpha_{1}^{1}+\alpha_{2}^{2}\right) \wedge \beta_{2}^{1}-\alpha_{1}^{2} \wedge \beta_{1}^{1}-\alpha_{2}^{1} \wedge \beta_{2}^{2} .
\end{array}\right.
$$




\subsection{Oriented Lagrangian planes}

Let $\Lambda_{2}^{+}$denote the set of oriented Lagrangian subspaces of $\mathbb{R}^{4}$, i.e. the set of oriented 2dimensional linear subspaces $V \subset \mathbb{R}^{4}$ such that $\Omega_{\mid V}=0$. The space $\Lambda_{2}^{+}$is a smooth manifold diffeomorphic to $S^{2} \times S^{1}$. The standard action of $\operatorname{Sp}(4, \mathbb{R})$ on $\mathbb{R}^{4}$ induces an action on $\Lambda_{2}^{+}$which is transitive. The projection map

$$
\pi_{\Lambda}: \mathbf{X} \in \operatorname{Sp}(4, \mathbb{R}) \mapsto\left[X_{1} \wedge X_{2}\right] \in \Lambda_{2}^{+}
$$

makes $\operatorname{Sp}(4, \mathbb{R})$ into a principal fiber bundle with structure group

$$
\operatorname{Sp}(4, \mathbb{R})_{1}=\left\{\mathbf{X}(A, b)=\left(\begin{array}{cc}
A & A b \\
0 & { }^{t} A^{-1}
\end{array}\right): \operatorname{det} A>0, b \in \mathrm{S}(2, \mathbb{R})\right\} .
$$

From this, it follows that the forms $\left(\gamma_{1}^{1}, \gamma_{2}^{2}, \gamma_{1}^{2}\right)$ span the semibasic forms of the projection $\pi_{\Lambda}$. Moreover, the symmetric quadratic form $g=-\gamma_{1}^{1} \gamma_{2}^{2}+\left(\gamma_{1}^{2}\right)^{2}$ and the exterior 3-form $\gamma_{1}^{1} \wedge \gamma_{1}^{2} \wedge \gamma_{2}^{2}$ are well defined on $\Lambda_{2}^{+}$, up to a positive multiple. They determine a conformal structure of signature $(2,1)$ and an orientation, respectively.

Remark 1. The group $\operatorname{Sp}(4, \mathbb{R})$ is a covering group of the identity component of the conformal Lorentz group $\mathrm{O}(3,2)$. This fact implies that $\Lambda_{2}^{+}$can be identified with the quotient of the nullcone of $\mathbb{R}^{3,2}$ by the action given by positive scalar multiplications. Therefore, $\Lambda_{2}^{+}$can be seen as the conformal compactification of oriented, time-oriented affine Minkowski space $\mathbb{R}^{2,1} \cong$ $\mathrm{S}(2, \mathbb{R})$ (cf. $[2,15])$. Any element of $\Lambda_{2}^{+}$can be represented by a $4 \times 2$ matrix

$$
X=\left(\begin{array}{c}
A_{1} \\
A_{2}
\end{array}\right)
$$

of rank 2 such that ${ }^{t} A_{1} A_{2}={ }^{t} A_{2} A_{1}$. Let $U_{0} \subset \Lambda_{2}^{+}$be the set of all Lagrangian subspaces $\left[X_{1} \wedge X_{2}\right]$ associated to ordered pairs of vectors

$$
X_{1}={ }^{t}\left(x_{1}^{1}, \ldots, x_{1}^{4}\right), \quad X_{2}={ }^{t}\left(x_{2}^{1}, \ldots, x_{2}^{4}\right)
$$

such that

$$
\operatorname{det}\left(\begin{array}{ll}
x_{1}^{1} & x_{2}^{1} \\
x_{1}^{2} & x_{2}^{2}
\end{array}\right)>0
$$

The set $U_{0}$ is a dense open subset of $\Lambda_{2}^{+}$. The map $S: U_{0} \rightarrow \mathrm{S}(2, \mathbb{R}) \cong \mathbb{R}^{2,1}$ given by

$$
\mathrm{S}\left(\left[X_{1} \wedge X_{2}\right]\right)=\left(\begin{array}{ll}
x_{1}^{3} & x_{2}^{3} \\
x_{1}^{4} & x_{2}^{4}
\end{array}\right)\left(\begin{array}{ll}
x_{1}^{1} & x_{2}^{1} \\
x_{1}^{2} & x_{2}^{2}
\end{array}\right)^{-1}
$$

is a conformal diffeomorphism, and $\left(U_{0}, S\right)$ is a local chart of $\Lambda_{2}^{+}$. Note that

$$
U_{0}=\left\{V \in \Lambda_{2}^{+}: V \cap W_{0}=\{0\}\right\},
$$

the set of Lagrangian subspaces which are transversal to $W_{0}=\operatorname{span}\left\{e_{3}, e_{4}\right\} \cdot{ }^{1}$ Let

$$
U_{1}=\left\{V \in \Lambda_{2}^{+}: \operatorname{dim}\left(V \cap W_{0}\right)=1\right\} .
$$

Then $U_{1} \cup\left\{W_{0}\right\}$ is a closed subset of $\Lambda_{2}^{+}$, which can be interpreted as the ideal boundary of $\mathbb{R}^{2,1} \cong U_{0}$. Note that $U_{0}$ and $U_{1}$ are orbits of the closed subgroup of $\operatorname{Sp}(4, \mathbb{R})$ that preserves the subspace $W_{0}$.

\footnotetext{
${ }^{1}$ Here $e_{1}, e_{2}, e_{3}, e_{4}$ denote the standard basis of $\mathbb{R}^{4}$.
} 


\subsection{Lagrangian immersions}

Let $f: M \rightarrow \mathbb{R}^{4}$ be a smooth immersion of a connected 2-manifold, oriented by a volume element $\mathcal{A}$. For any $q \in M$, let $\mathcal{T}_{f}(q)$ be the 2-plane $d f\left(T_{q} M\right)$ translated to the origin and equipped with the orientation induced by $-d f\left(\left.\mathcal{A}\right|_{q}\right) .^{2}$

Definition 1. The immersion $f: M \rightarrow \mathbb{R}^{4}$ is called Lagrangian if $\mathcal{T}_{f}(q) \in \Lambda_{2}^{+}$, for each $q \in M$. The resulting map

$$
\mathcal{T}_{f}: q \in M \mapsto \mathcal{T}_{f}(q) \in \Lambda_{2}^{+}
$$

is the (symplectic) Gauss map of the Lagrangian immersion $f$.

Definition 2. A Lagrangian immersion is said of general type (respectively, special type) if its Gauss map is an immersion (respectively, has constant rank one). Moreover, a Lagrangian immersion of general type is said elliptic (respectively, hyperbolic, parabolic) if its Gauss map is a spacelike (respectively, timelike, lightlike) immersion into $\Lambda_{2}^{+}$.

\section{Moving frames on elliptic Lagrangian immersions}

\subsection{Frame reductions and differential invariants}

In this section, $f: M \rightarrow \mathbb{R}^{4}$ will denote an elliptic Lagrangian immersion with Gauss map $\mathcal{T}_{f}$. Since we are working with immersions which are not necessarily one-to-one, it is not restrictive to assume that $M$ is simply connected.

Definition 3. A symplectic frame field along $f$ is a smooth map

$$
\mathbf{S}=\mathbf{S}(f, \mathbf{X}): U \rightarrow \mathcal{S}(4, \mathbb{R})
$$

from an open connected subset $U \subset M$ such that the projection (1) composed with $\mathbf{S}$ is $f$, i.e. $\mathbf{P} \circ \mathbf{S}(f, \mathbf{X})=\mathbf{S}(f, \mathbf{X}) \cdot O=f$.

Following the usual practice in the method of moving frames we will write $\phi$ instead of $\mathbf{S}^{*}(\phi)$ to denote forms on $U$ which are pulled back from $\mathcal{S}(4, \mathbb{R})$ by the moving frame $\mathbf{S}$. A symplectic frame field along $f$ gives a local representation of the derivative map,

$$
d f=\sum_{j=1}^{4} \tau^{j} \mathrm{X}_{j} .
$$

Let $\mathbf{S}: U \rightarrow \mathcal{S}(4, \mathbb{R})$ be a symplectic frame field along $f$. Any other symplectic frame field along $f$ on $U$ is given by

$$
\widetilde{\mathbf{S}}=\mathbf{S Y}
$$

where $\mathbf{Y}: U \rightarrow \operatorname{Sp}(4, \mathbb{R})$ is a smooth map $^{3}$. If $\Theta$ and $\widetilde{\Theta}$ are the pull-backs of the Maurer-Cartan form of $\mathcal{S}(4, \mathbb{R})$ by $\mathbf{S}$ and $\widetilde{\mathbf{S}}$, respectively, then

$$
\widetilde{\Theta}=\mathbf{Y}^{-1} \Theta \mathbf{Y}+\mathbf{Y}^{-1} d \mathbf{Y}
$$

\footnotetext{
${ }^{2}$ One reason for choosing the opposite orientation is that totally umbilic elliptic Lagrangian immersions are then described in terms of holomorphic functions instead of anti-holomorphic ones (cf. Sections 4.4 and 4.5 ).

${ }^{3}$ Here we are identifying an element $\mathbf{S}(0, \mathbf{Y}) \in \mathcal{S}(4, \mathbb{R})$ with $\mathbf{Y} \in \operatorname{Sp}(4, \mathbb{R})$, and hence an element $\mathrm{S}(0, \mathbf{x}) \in \mathfrak{s}(4, \mathbb{R})$ with $\mathbf{x} \in \mathfrak{s p}(4, \mathbb{R})$.
} 


\subsubsection{First order frame fields}

A symplectic frame field $\mathbf{S}=\mathbf{S}(f, \mathbf{X}): U \rightarrow \mathcal{S}(4, \mathbb{R})$ along $f$ is of first order if

$$
\mathcal{T}_{f}(q)=\left[\left.\left.\mathrm{X}_{1}\right|_{q} \wedge \mathrm{X}_{2}\right|_{q}\right], \quad \text { for each } q \in U .
$$

It is clear that first order frame fields exist locally. With respect to a first order frame, the 1-forms $\tau^{3}$ and $\tau^{4}$ vanish identically on $U$ and

$$
d f=\tau^{1} \mathrm{X}_{1}+\tau^{2} \mathrm{X}_{2},
$$

where $\tau^{1} \wedge \tau^{2}<0$. If $\mathbf{S}$ is a first order frame field along $f$ on $U$, then any other is given by $\widetilde{\mathbf{S}}=\mathbf{S Y}(A, b)$, where $\mathbf{Y}(A, b): U \rightarrow \operatorname{Sp}(4, \mathbb{R})_{1}$ is a smooth map into the subgroup $\operatorname{Sp}(4, \mathbb{R})_{1}$ introduced in Section 2.2.

Calculated with respect to a first order frame field, the quadratic form $g=-\gamma_{1}^{1} \gamma_{2}^{2}+\left(\gamma_{1}^{2}\right)^{2}$ is positive definite. Under a change $\widetilde{\mathbf{S}}=\mathbf{S Y}(A, b)$ of first order frames, it transforms by

$$
-\widetilde{\gamma}_{1}^{1} \widetilde{\gamma}_{2}^{2}+\left(\widetilde{\gamma}_{1}^{2}\right)^{2}=\operatorname{det}(A)^{2}\left(-\gamma_{1}^{1} \gamma_{2}^{2}+\left(\gamma_{1}^{2}\right)^{2}\right),
$$

and hence defines a conformal structure on $M$. On $M$, we will consider the unique complex structure compatible with the given orientation and the conformal structure defined by $g$. The 1-forms

$$
\omega^{1}:=\frac{1}{2}\left(\gamma_{1}^{1}-\gamma_{2}^{2}\right), \quad \omega^{2}:=\gamma_{1}^{2}
$$

are everywhere linearly independent and

$$
\omega=\omega^{1}+i \omega^{2}
$$

is complex-valued of bidegree $(1,0)$. Moreover, there exist smooth functions $\ell_{1}, \ell_{2}: U \rightarrow \mathbb{R}$ such that

$$
\frac{1}{2}\left(\gamma_{1}^{1}+\gamma_{2}^{2}\right)=\ell_{1} \omega^{1}+\ell_{2} \omega^{2}, \quad \ell_{1}^{2}+\ell_{2}^{2}<1 .
$$

\subsubsection{Second order frame fields}

A first order frame field along $f$ is of second order if it satisfies

$$
\frac{1}{2}\left(\gamma_{1}^{1}+\gamma_{2}^{2}\right)=0
$$

Lemma 1. About any point of $M$ there exists a second order frame field along $f$.

Proof. Let $\mathbf{S}: U \rightarrow \mathcal{S}(4, \mathbb{R})$ be any first order frame field along $f$. Let $\phi: U \rightarrow(-\pi / 2, \pi / 2)$ be the smooth function defined by

$$
\sin (\phi)=-\frac{\ell_{2}}{\sqrt{1-\ell_{1}^{2}}}
$$

and let $A: U \rightarrow \mathrm{GL}_{+}(2, \mathbb{R})$ be given by

$$
A=\left(\begin{array}{cc}
\sqrt{\frac{1-\ell_{1}}{2}} \cos (\phi) & \sqrt{\frac{1-\ell_{1}}{2}} \sin (\phi) \\
0 & \sqrt{\frac{1+\ell_{1}}{2}}
\end{array}\right) .
$$

Then $\mathbf{Y}(A, 0): U \rightarrow \mathbf{S p}(4, \mathbb{R})_{1}$ is a smooth map. Let $\widetilde{\mathbf{S}}=\mathbf{S Y}(A, 0)$. The transformation rule (4) gives $\widetilde{\gamma}={ }^{t} A \gamma A$, from which we compute $\widetilde{\gamma}_{1}^{1}+\widetilde{\gamma}_{2}^{2}=0$. This means that $\widetilde{\mathbf{S}}$ is a second order frame field along $f$. 
If $\mathbf{S}$ and $\widetilde{\mathbf{S}}$ are second order frame fields on $U$, then $\widetilde{\mathbf{S}}=\mathbf{S Y}(A, b)$, where $A$ is a $2 \times 2$ matrix such that ${ }^{t} A A=r^{2} I_{2}$ and $\operatorname{det}(A)>0$. The group of all such $A$ identifies with the multiplicative group $\mathbb{C}^{*}$ of nonzero complex numbers by

$$
\mathbb{C}^{*} \ni r e^{i s} \mapsto r\left(\begin{array}{cc}
\cos (s) & -\sin (s) \\
\sin (s) & \cos (s)
\end{array}\right) .
$$

Let $\mathbf{S}$ be a second order frame field along $f$. We know that the complex-valued 1-form $\omega=$ $\omega^{1}+i \omega^{2}$ is of bidegree $(1,0)$ and never zero.

Differentiating the equations $\tau^{3}=\tau^{4}=0$ and using the structure equations, we find that

$$
\left\{\begin{array}{l}
\tau^{1} \wedge \omega^{1}+\tau^{2} \wedge \omega^{2}=0 \\
\tau^{1} \wedge \omega^{2}-\tau^{2} \wedge \omega^{1}=0
\end{array}\right.
$$

which, by Cartan's Lemma, implies that

$$
\tau:=\tau^{1}-i \tau^{2}=t \omega
$$

for some smooth function $t=t_{1}+i t_{2}: U \rightarrow \mathbb{C}$. The 1 -form $\tau$ is of bidegree $(1,0)$ and never zero.

Under a change of second order frame fields $\widetilde{\mathbf{S}}=\mathbf{S Y}\left(r e^{i s}, b\right)$, the forms $\omega$ and $\tau$ transform by

$$
\widetilde{\omega}=r^{2} e^{-2 i s} \omega, \quad \widetilde{\tau}=r^{-1} e^{i s} \tau .
$$

This implies that

$$
\widetilde{\tau}^{2} \widetilde{\omega}=\tau^{2} \omega .
$$

Thus $\tau^{2} \omega$ is independent of the choice of second order frame field and is never zero.

Definition 4. The cubic differential form $\mathcal{F}:=\tau^{2} \omega$, globally defined on $M$, is called the Fubini cubic form of the Lagrangian immersion $f$.

Next, consider the complex-valued 1-form

$$
\eta:=\eta_{1}-i \eta_{2}=\frac{1}{2}\left(\alpha_{1}^{1}-\alpha_{2}^{2}\right)-\frac{i}{2}\left(\alpha_{1}^{2}+\alpha_{2}^{1}\right) .
$$

Differentiation of $\gamma_{1}^{1}+\gamma_{2}^{2}=0$, combined with the structure equations, gives

$$
\eta_{1} \wedge \omega^{1}+\eta_{2} \wedge \omega^{2}=0
$$

which, by Cartan's Lemma, implies that

$$
\eta=h \omega+\ell \bar{\omega}
$$

for some smooth functions $h=h_{1}+i h_{2}: U \rightarrow \mathbb{C}$ and $\ell: U \rightarrow \mathbb{R}$.

\subsubsection{Third order frame fields}

Under a change $\widetilde{\mathbf{S}}=\mathbf{S Y}\left(r e^{i s}, b\right)$ of second order frame fields, the 1-form $\eta$ transforms by

$$
\widetilde{\eta}=e^{2 i s}\left(\eta-\frac{r^{2}}{2} \operatorname{tr}(b) \bar{\omega}\right) .
$$

This implies that locally there exist second order frame fields satisfying $\ell=0$. Such frame fields are said of third order.

If $\widetilde{\mathbf{S}}$ and $\mathbf{S}$ are third order frame fields on $U$, then $\widetilde{\mathbf{S}}=\mathbf{S Y}\left(r e^{i s}, b\right)$ where $\operatorname{tr}(b)=0$. With respect to a third order frame field, the 1-form $\eta$ is of bidegree $(1,0)$ and transforms by

$$
\widetilde{\eta}=e^{2 i s} \eta .
$$


Definition 5. According to (5) and (6), the differential forms

$$
\mathcal{B}=\bar{\eta} \eta, \quad \mathcal{H}=|t|^{2 / 3} \eta \omega
$$

are globally defined on $M$, independent of the choice of third order frames. We call $\mathcal{B}$ the Thomsen quadratic form and $\mathcal{H}$ the normalized Hopf differential of $f$.

Remark 2. The invariant form $\mathcal{B}$ amounts to the metric induced on $M$ by the conformal Gauss map [5] (or central sphere congruence [4]) of the spacelike conformal immersion $\mathcal{T}_{f}: M \rightarrow \Lambda_{2}^{+}$. The invariant form $\mathcal{H}$ is instead a normalization of the Hopf differential of $\mathcal{T}_{f}$.

\subsection{Adapted frame fields}

Being simply connected, $M$ is either the Riemann sphere $S^{2}$, the complex plane $\mathbb{C}$, or the unit disk $\Delta$. By a complex parameter on $M$ is meant a complex coordinate chart $(U, z)$ in $M$ defined on a maximal contractible open subset $U$ of $M$ : if $M=\mathbb{C}$, or $\Delta$, then $U=M$; if $M=S^{2}$, then $U=S^{2} \backslash\{q\}$.

Definition 6. Let $(U, z)$ be a complex parameter on $M$. A third order frame field $\mathbf{S}: U \rightarrow$ $\mathcal{S}(4, \mathbb{R})$ along $f$ is adapted to $(U, z)$ if

$$
\omega=\omega^{1}+i \omega^{2}=d z, \quad \alpha_{1}^{1}+\alpha_{2}^{2}=\alpha_{1}^{2}-\alpha_{2}^{1}=0 .
$$

Proposition 1. Let $(U, z)$ be a complex parameter on $M$. There exist third order frame fields along $f$ adapted to $(U, z)$. If $\mathbf{S}$ and $\mathbf{S}^{\prime}$ are two such frame fields, then $\mathbf{S}^{\prime}= \pm \mathbf{S}$.

Proof. Let $q_{0} \in U$ and let $\mathbf{S}^{\prime}: V \rightarrow \mathcal{S}(4, \mathbb{R})$ be any third order frame field defined on a simply connected open neighborhood $V \subset U$ of $q_{0}$. On $V$ there exist real-valued functions $r, \vartheta: V \rightarrow \mathbb{R}$ such that $\omega^{\prime}=r^{2} e^{-2 i \vartheta} d z$. Define $\mathbf{S}^{\prime \prime}=\mathbf{S}^{\prime} \mathbf{Y}\left(r^{-2} e^{2 i \vartheta}, 0\right)$. Then, $\mathbf{S}^{\prime \prime}$ is a third order frame field such that $\omega^{\prime \prime}=d z$. Two such frame fields are related by

$$
\widetilde{\mathbf{S}}^{\prime \prime}=\mathbf{S}^{\prime \prime} \mathbf{Y}\left( \pm I_{2}, b\right)
$$

where $b: V \rightarrow \mathrm{S}(2, \mathbb{R})$ is a smooth map such that $\operatorname{tr}(b)=0$. From the structure equations we find that

$$
\left\{\begin{array}{l}
\left(\alpha_{1}^{1}+\alpha_{2}^{2}\right) \wedge d x+\left(\alpha_{1}^{2}-\alpha_{2}^{1}\right) \wedge d y=0 \\
\left(\alpha_{1}^{1}+\alpha_{2}^{2}\right) \wedge d y-\left(\alpha_{1}^{2}-\alpha_{2}^{1}\right) \wedge d x=0
\end{array}\right.
$$

which implies

$$
\left(\alpha_{1}^{1}+\alpha_{2}^{2}\right)-i\left(\alpha_{1}^{2}-\alpha_{2}^{1}\right)=w d z
$$

for some smooth function $w: V \rightarrow \mathbb{C}$. If $\widetilde{\mathbf{S}}^{\prime \prime}=\mathbf{S}^{\prime \prime} \mathbf{Y}\left( \pm I_{2}, b\right)$ then

$$
\left(\tilde{\alpha}_{1}^{1}+\tilde{\alpha}_{2}^{2}\right)-i\left(\tilde{\alpha}_{1}^{2}-\tilde{\alpha}_{2}^{1}\right)=\left(\alpha_{1}^{1}+\alpha_{2}^{2}\right)-i\left(\alpha_{1}^{2}-\alpha_{2}^{1}\right)+2\left(b_{1}^{1}-i b_{1}^{2}\right) d z .
$$

If we choose $b$ such that $2\left(b_{1}^{1}-i b_{1}^{2}\right)=-w$, then $\widetilde{\mathbf{S}}^{\prime \prime}$ satisfies

$$
\omega^{\prime \prime}=d z, \quad\left(\tilde{\alpha}_{1}^{1}+\tilde{\alpha}_{2}^{2}\right)-i\left(\tilde{\alpha}_{1}^{2}-\tilde{\alpha}_{2}^{1}\right)=0 .
$$

This shows that adapted frame fields do exist locally near any point of $U$. Moreover, two such frames $\mathbf{S}$ and $\mathbf{S}^{\prime}$ on $V$ are related by $\mathbf{S}^{\prime}= \pm \mathbf{S}$. The existence of an adapted frame on $U$ follows from the fact that $U$ is simply connected. 


\subsubsection{Invariant functions and integrability equations}

Let $\mathbf{S}: U \rightarrow \mathcal{S}(4, \mathbb{R})$ be a frame field adapted to $(U, z)$. Then

$$
\tau=t d z, \quad \eta=h d z,
$$

for smooth functions $t=t_{1}+i t_{2}: U \rightarrow \mathbb{C}$ and $h=h_{1}+i h_{2}: U \rightarrow \mathbb{C}$. Define $v$ and $\rho$ by

$$
v:=\frac{1}{2}\left(\beta_{1}^{1}+\beta_{2}^{2}\right), \quad \rho:=\frac{1}{2}\left(\beta_{1}^{1}-\beta_{2}^{2}\right)-i \beta_{1}^{2} .
$$

The structure equations (2) imply

$$
v=h_{\bar{z}} d z+\bar{h}_{z} d \bar{z},
$$

and

$$
\rho=p d z+\left(h_{1}^{2}+h_{2}^{2}\right) d \bar{z}=p d z+|h|^{2} d \bar{z}
$$

for some smooth function $p=p_{1}+i p_{2}: U \rightarrow \mathbb{C}$.

Definition 7. The functions $t, h$ and $p$ defined by (8) and (10) are called the invariant functions of the frame field along $f$ adapted to $(U, z)$. These functions are subject to the equations

$$
\left\{\begin{array}{l}
t_{\bar{z}}=\bar{t} h, \\
p_{\bar{z}}=2 h \bar{h}_{z}-i|h|_{z}^{2}, \\
\bar{p} h-p \bar{h}=h_{\bar{z} \bar{z}}-\bar{h}_{z z} .
\end{array}\right.
$$

Remark 3. From (11) it follows that

$$
\bar{\partial}(\mathcal{F})=2 \mathcal{N},
$$

where $\mathcal{N}:=\bar{\tau} \tau \eta \omega$ is an invariant form. The form $\mathcal{B}, \mathcal{H}$ and $\mathcal{N}$ are completely determined by the Fubini cubic form.

Theorem 1 (Existence). Let $(U, z)$ be a complex parameter on $M$. Let $t: U \rightarrow \mathbb{C}, h$ : $U \rightarrow \mathbb{C}$ and $p: U \rightarrow \mathbb{C}$ be smooth functions satisfying the equations (11). Then there exist an elliptic Lagrangian immersion $f: U \rightarrow \mathbb{R}^{4}$, unique up to affine symplectic transformation, and a unique frame field $\mathbf{S}: U \rightarrow \mathcal{S}(4, \mathbb{R})$ along $f$ adapted to $(U, z)$ whose invariant functions are $t, h$ and $p$, i.e.

$$
\tau=t d z, \quad \eta=h d z, \quad \rho^{(1,0)}=p d z .
$$

Proof. Let

$$
\begin{aligned}
& \left(\begin{array}{l}
\tau^{1} \\
\tau^{2}
\end{array}\right)=\left(\begin{array}{c}
t_{1} d x-t_{2} d y \\
-t_{2} d x-t_{1} d y
\end{array}\right), \quad \gamma=\left(\begin{array}{cc}
d x & d y \\
d y & -d x
\end{array}\right), \\
& \alpha=\left(\begin{array}{cc}
h_{1} d x-h_{2} d y & -h_{2} d x-h_{1} d y \\
-h_{2} d x-h_{1} d y & -h_{1} d x+h_{2} d y
\end{array}\right), \quad \beta=\left(\begin{array}{ll}
\beta_{1}^{1} & \beta_{1}^{2} \\
\beta_{1}^{2} & \beta_{2}^{2}
\end{array}\right),
\end{aligned}
$$

where

$$
\frac{1}{2}\left(\beta_{1}^{1}+\beta_{2}^{2}\right)=h_{\bar{z}} d z+\bar{h}_{z} d \bar{z}
$$

and

$$
\frac{1}{2}\left(\beta_{1}^{1}-\beta_{2}^{2}\right)-i \beta_{1}^{2}=p d z+|h|^{2} d \bar{z} .
$$


Then

$$
\Theta=\left(\begin{array}{ccc}
0 & 0 & 0 \\
\left(\begin{array}{c}
\tau^{1} \\
\tau^{2}
\end{array}\right) & \alpha & \beta \\
0 & \gamma & -{ }^{t} \alpha
\end{array}\right)
$$

is a smooth 1 -form on $U$ with values in $\mathfrak{s}(4, \mathbb{R})$. By $(11)$,

$$
d \Theta=-\Theta \wedge \Theta
$$

Therefore, by the Cartan-Darboux theorem, there exists a smooth map $\mathbf{S}: U \rightarrow \mathcal{S}(4, \mathbb{R})$ such that $\mathbf{S}^{-1} d \mathbf{S}=\Theta$, unique up to left multiplication by an element of $\mathcal{S}(4, \mathbb{R})$. The elliptic Lagrangian immersion $f: U \rightarrow \mathbb{R}^{4}$ defined by $f=\mathbf{S} \cdot O$ has the required properties.

Remark 4 (Uniqueness). If $f, \tilde{f}: U \rightarrow \mathbb{R}^{4}$ are two elliptic Lagrangian immersions inducing the same invariant functions, then, by the Cartan-Darboux uniqueness theorem, there exists a symplectic motion $\mathbf{S}: \mathbb{R}^{4} \rightarrow \mathbb{R}^{4}$ such that $\tilde{f}=\mathbf{S} f$.

\section{Symplectic applicability}

In this section we investigate the extent to which the invariants are really needed to determine an elliptic Lagrangian immersion up to symmetry. We take the point of view of surface applicability as in classical projective differential geometry and Lie sphere geometry [12, 8, 9, 4, 23, 18].

\subsection{Generic Lagrangian immersions}

Suppose that $\mathcal{H}$, the normalized Hopf differential, is never zero. Then the Hermitian form

$$
\mathcal{P}=\left(\left(\bar{h}_{z} \bar{h}^{-1}\right)_{\bar{z}}-\left(h_{\bar{z}} h^{-1}\right)_{z}\right) d z d \bar{z}
$$

is well defined, independent of the choice of the complex parameter $(U, z)$ and of the adapted frame (the reason for considering this form will be clear in the proof of the next proposition).

Definition 8. An elliptic Lagrangian immersion $f: M \rightarrow \mathbb{R}^{4}$ is called generic if $\mathcal{H}(q) \neq 0$ and $\mathcal{P}(q) \neq 0$, for each $q \in M$.

Proposition 2. A generic elliptic Lagrangian immersion is uniquely determined, up to affine symplectic transformation, by the Fubini cubic form.

Proof. Fix a complex parameter $(U, z)$ and choose an adapted frame field $\mathbf{S}: U \rightarrow \mathcal{S}(4, \mathbb{R})$. If $h \neq 0$, then (11) implies the existence of a unique real-valued function $s: U \rightarrow \mathbb{R}$ such that

$$
p=h s+\mathcal{D}_{2}(h),
$$

where

$$
\mathcal{D}_{2}(h)=\frac{1}{2 \bar{h}}\left(\bar{h}_{z z}-h_{\bar{z} \bar{z}}\right) .
$$

Differentiating this identity and using

$$
p_{\bar{z}}=2 h \bar{h}_{z}-i|h|_{z}^{2}
$$


we get

$$
d s=-s\left(\frac{1}{h} h_{\bar{z}} d \bar{z}+\frac{1}{\bar{h}} \bar{h}_{z} d z\right)-\left(\mathcal{D}_{3}(h) d \bar{z}+\overline{\mathcal{D}_{3}(h)} d z\right),
$$

where

$$
\mathcal{D}_{3}(h)=\frac{1}{h}\left[\left(\mathcal{D}_{2}(h)\right)_{\bar{z}}-2 h \bar{h}_{z}+i|h|_{z}^{2}\right] .
$$

Differentiation of (13) gives

$$
\mathcal{P}_{2}(h) s+\mathcal{D}_{4}(h)=0,
$$

where

$$
\mathcal{P}_{2}(h)=\left(\bar{h}_{z} \bar{h}^{-1}\right)_{\bar{z}}-\left(h_{\bar{z}} h^{-1}\right)_{z}
$$

and

$$
\mathcal{D}_{4}(h)={\overline{\mathcal{D}_{3}(h)_{\bar{z}}}}_{\bar{D}}-\mathcal{D}_{3}(h)_{z}-\bar{h}^{-1} \bar{h}_{z} \mathcal{D}_{3}(h)+h^{-1} h_{\bar{z}} \overline{\mathcal{D}_{3}(h)} .
$$

If $\mathcal{P}_{2}(h) \neq 0$, then

$$
s=-\frac{\mathcal{D}_{4}(h)}{\mathcal{P}_{2}(h)} .
$$

Therefore, for a generic elliptic Lagrangian immersion (i.e. $h \neq 0$ and $\mathcal{P}_{2}(h) \neq 0$ ), the functions $h$ and $p$ are determined by the Fubini cubic form. Thus, a generic $f$ is uniquely determined, up to symplectic congruence, by its Fubini cubic form.

\subsection{Applicable elliptic Lagrangian immersions}

In this section we investigate the special class of surfaces which are not determined by the Fubini cubic form alone.

Definition 9. Two noncongruent elliptic Lagrangian immersions $f, \tilde{f}: M \rightarrow \mathbb{R}^{4}$ are applicable if they induce the same Fubini cubic forms, $\mathcal{F}=\widetilde{\mathcal{F}}$. An elliptic Lagrangian immersion $f: M \rightarrow \mathbb{R}^{4}$ is applicable if there exists an elliptic Lagrangian immersion $\widetilde{f}: M \rightarrow \mathbb{R}^{4}$ such that $f$ and $\tilde{f}$ are applicable. Moreover, an elliptic Lagrangian immersion $f$ is rigid if any elliptic Lagrangian immersion inducing the same Fubini cubic form as that of $f$ is congruent to $f$.

Remark 5. According to Proposition 2, any generic elliptic Lagrangian immersion is rigid.

Theorem 2. An elliptic Lagrangian immersion $f: M \rightarrow \mathbb{R}^{4}$ is applicable if and only if there exists a non-zero holomorphic quadratic differential $\mathcal{Q}$ on $M$ such that $\mathcal{H} \wedge_{\mathbb{R}} \mathcal{Q}=0 .{ }^{4}$

Proof. First, we consider the case $\mathcal{H} \equiv 0$. Then, $\mathcal{F}$ is a never vanishing holomorphic cubic differential. Since $M$ is simply connected, $M$ is either the complex plane or the unit disk. In both cases, there exist a global complex coordinate $z: M \rightarrow \mathbb{C}$ and a global adapted frame field $\mathbf{S}: M \rightarrow \mathcal{S}(4, \mathbb{R})$ with invariant functions $t: M \rightarrow \mathbb{C}, h=0$ and $p: M \rightarrow \mathbb{C}$. Consider a non-zero holomorphic differential $\mathcal{Q}=\lambda d z^{2}$ and set $\widetilde{p}=p-\lambda$. The functions $t, h=0$ and $\widetilde{p}$ satisfy equations (11). The corresponding $\widetilde{f}$ has $\widetilde{\mathcal{F}}=\mathcal{F}$, but is not congruent to $f$.

We now examine the case $\mathcal{H} \neq 0$. Let $f, \widetilde{f}$ be two noncongruent elliptic Lagrangian immersions inducing the same Fubini cubic forms. Let $\mathbf{S}, \widetilde{\mathbf{S}}: U \rightarrow \mathcal{S}(4, \mathbb{R})$ be the corresponding

\footnotetext{
${ }^{4} \mathcal{H} \wedge \mathbb{R} \mathcal{Q}=0$ means that $\mathcal{H}$ and $\mathcal{Q}$ are linearly dependent over the reals.
} 
adapted frame fields with respect to a fixed complex parameter $(U, z)$. Since $t=\widetilde{t}$, equations (11) imply that $h=\widetilde{h}$ and that $p-\widetilde{p}$ is a non-zero holomorphic function satisfying

$$
\left(p_{1}-\widetilde{p}_{1}\right) h_{2}-\left(p_{2}-\widetilde{p}_{2}\right) h_{1}=0 .
$$

Then, $\mathcal{Q}_{U}=(p-\widetilde{p}) d z^{2}$ is a non-zero holomorphic quadratic differential on $U$ such that $\mathcal{H} \wedge_{\mathbb{R}} \mathcal{Q}_{U}=$ 0 . Let $(\widehat{U}, \widehat{z})$ be another complex parameter and let $\widehat{\mathcal{Q}}_{\hat{U}}$ be the corresponding holomorphic quadratic differential. The equations $\mathcal{H} \wedge_{\mathbb{R}} \mathcal{Q}_{U}=0$ and $\mathcal{H} \wedge_{\mathbb{R}} \widehat{\mathcal{Q}}_{\hat{U}}=0$ imply that $\mathcal{Q}=\lambda_{U \hat{U}} \widehat{Q}$ on $U \cap \widehat{U} \neq \varnothing$, where $\lambda_{U \hat{U}}$ is a real-valued, locally constant function. The functions $\left\{\exp \lambda_{U \hat{U}}\right\}$, defined on each ordered pair $(U, \widehat{U})$ when $U \cap \widehat{U} \neq \varnothing$, generate an $\mathbb{R}^{+}$-valued 1-cocycle on $M$, which is trivial because $M$ is simply connected. Therefore, there exists a globally defined nonzero holomorphic quadratic differential $\mathcal{Q}^{\prime}$ such that $\mathcal{Q}_{U}=\left.\mathcal{Q}^{\prime}\right|_{U}$, for every $(U, z)$.

Conversely, suppose there exists a non-zero holomorphic quadratic differential $\mathcal{Q}$ such that $\mathcal{H} \wedge_{\mathbb{R}} \mathcal{Q}=0$. This implies that $M$ is either $\mathbb{C}$ or the unit disk. Let $z$ be a global complex coordinate on $M$ and let $\mathbf{S}: M \rightarrow \mathcal{S}(4, \mathbb{R})$ be the associated adapted frame field. Set $\mathcal{Q}=w d z^{2}$, where $w=w_{1}+i w_{2}$ is a holomorphic non-zero function. Now, the invariant functions $t, h$ and $p$, calculated with respect to $\mathbf{S}$, satisfy (11). Since $\mathcal{H} \wedge_{\mathbb{R}} \mathcal{Q}=0$, that is $w_{1} h_{2}-w_{2} h_{1}=0$, also $t, h$ and $\widetilde{p}=p-w$ satisfy (11). The corresponding elliptic Lagrangian immersions $f, \widetilde{f}: M \rightarrow \mathbb{R}^{4}$ are applicable.

Remark 6. If $\mathcal{H} \neq 0$, then equation $\mathcal{H} \wedge_{\mathbb{R}} \mathcal{Q}=0$ determines $\mathcal{Q}$ up to a real constant multiple. For each $\lambda \in \mathbb{R}$, define the invariant functions $t_{\lambda}=t, h_{\lambda}=h$ and $p_{\lambda}=p-\lambda$. It is clear that $t_{\lambda}, h_{\lambda}$ and $p_{\lambda}$ satisfy the integrability equations (11). Therefore, up to affine symplectic transformation, there exists a unique $f_{\lambda}$ with $\mathcal{F}_{\lambda}=\mathcal{F}$. As $p_{\lambda}$ are distinct for distinct values of $\lambda$, the immersions $f_{\lambda}$ are noncongruent for distinct values of $\lambda$. This shows that applicable elliptic Lagrangian immersions come in 1-parameter families.

Interestingly enough, away from the points where $h$ vanishes (umbilics), the Gauss map of an applicable elliptic Lagrangian immersion $f$ is an isothermic spacelike immersion. Moreover, the Gauss maps of the 1-parameter family of associates to $f$ are the $T$-transforms of Bianchi and Calapso of $\gamma_{f}$ (cf. $\left.[7,3,16]\right)$.

Remark 7. The 1-parameter family of associates to an applicable elliptic Lagrangian immersion are related to its second order affine symplectic deformations. We will not discuss this issue here. We just recall that two elliptic Lagrangian immersions $f, \tilde{f}: M \rightarrow \mathbb{R}^{4}$ are second order affine symplectic deformations of each other if there exists a smooth map $D: M \rightarrow \mathcal{S}(4, \mathbb{R})$ such that, for each $q \in M$, the Taylor expansions about $q$ of $D(q) f$ and $\widetilde{f}$ agree through second order terms. For the general notion of applicability and deformation in homogeneous spaces we refer the reader to $[14,17]$.

\subsection{The differential equations of applicable Lagrangian immersions}

Let $f: M \rightarrow \mathbb{R}^{4}$ be an applicable elliptic Lagrangian immersion and let $\mathcal{Q}$ be a non-zero holomorphic quadratic differential on $M$ such that $\mathcal{H} \wedge_{\mathbb{R}} \mathcal{Q}=0$. If we suppose that $\mathcal{Q}$ never vanishes, we can choose a complex coordinate $(U, z)$ such that $\mathcal{Q}=d z^{2}$. Let $\mathbf{S}: U \rightarrow \mathcal{S}(4, \mathbb{R})$ be a frame field along $f$ adapted to $(U, z)$ with invariant functions $t, h$ and $p$. Now, the equation $\mathcal{H} \wedge_{\mathbb{R}} \mathcal{Q}=0$ implies that $h$ is real valued.

Next, in analogy with $[6,24]$ we deduce the differential equations satisfied by the invariant functions of an elliptic Lagrangian immersion. With respect to the adapted frame field $\mathbf{S}$, we have

$$
\alpha_{1}^{1}=-\alpha_{2}^{2}=h d x, \quad \alpha_{1}^{2}=\alpha_{2}^{1}=-h d y,
$$


where $h$ is a real-valued function. From (2), we compute

$$
\left\{\begin{array}{l}
\beta_{1}^{1}=\left(p_{1}+h_{x}+h^{2}\right) d x-\left(p_{2}+h_{y}\right) d y \\
\beta_{2}^{2}=-\left(p_{1}-h_{x}+h^{2}\right) d x+\left(p_{2}-h_{y}\right) d y \\
\beta_{1}^{2}=-p_{2} d x-\left(p_{1}-h^{2}\right) d y
\end{array}\right.
$$

Using (3), we find

$$
\left\{\begin{array}{l}
h_{x y}=-2 h p_{2} \\
\left(p_{1}\right)_{y}=-\left(p_{2}\right)_{x}-2\left(h^{2}\right)_{y} \\
\left(p_{1}\right)_{x}=\left(p_{2}\right)_{y}+2\left(h^{2}\right)_{x} .
\end{array}\right.
$$

Thus, calculated with respect to a complex parameter $z$ such that $\mathcal{Q}=d z^{2}$, the integrability equations of an applicable elliptic Lagrangian immersion are

$$
\left\{\begin{array}{l}
t_{\bar{z}}=h \bar{t} \\
h_{x y}=-2 h p_{2}, \\
\Delta p_{2}=-4\left(h^{2}\right)_{x y}
\end{array}\right.
$$

If $\left(t, h, p_{2}\right)$ is a solution of this system, the 1 -form

$$
\left[\left(p_{2}\right)_{y}+2\left(h^{2}\right)_{x}\right] d x-\left[\left(p_{2}\right)_{x}+2\left(h^{2}\right)_{y}\right] d y
$$

is closed. If $p_{1}: M \rightarrow \mathbb{R}$ is so that

$$
d p_{1}=\left[\left(p_{2}\right)_{y}+2\left(h^{2}\right)_{x}\right] d x-\left[\left(p_{2}\right)_{x}+2\left(h^{2}\right)_{y}\right] d y
$$

then $t, h$ and $p=p_{1}+i p_{2}$ give a solution of (11). Thus, up to congruence, there exists a unique elliptic Lagrangian immersion $f: M \rightarrow \mathbb{R}^{4}$ with invariant functions $t, h$ and $p$. Different choices of the primitive $p_{1}$ yield the family of associate immersions applicable to $f$.

\subsection{Totally umbilic Lagrangian immersions}

Definition 10. An elliptic Lagrangian immersion $f$ is called totally umbilic if its Hopf differential $\mathcal{H}$ vanishes identically, or, equivalently, $h$ vanishes identically. This terminology is based on the observation that $\mathcal{H} \equiv 0$ if and only if the Gauss map $\mathcal{T}_{f}$ of $f$ is a totally umbilic spacelike immersion (cf. [5]).

Remark 8. Note that $f$ is totally umbilic if and only if its Fubini cubic form $\mathcal{F}$ is holomorphic (cf. (12)). In this case, $M$ is either the complex plane or the unit disk. Therefore, there exists a global parameter $z$ on $M$ and a global adapted frame field $\mathbf{S}: M \rightarrow \mathcal{S}(4, \mathbb{R})$ along $f$.

Proposition 3. Let $f, \tilde{f}: M \rightarrow \mathbb{R}^{4}$ be two noncongruent, totally umbilic elliptic Lagrangian immersions. Then there exists a biholomorphism $\Phi: M \rightarrow M$ such that $f$ and $\tilde{f} \circ \Phi$ are applicable.

Proof. On $M$ there are complex parameters $z, w: M \rightarrow W, W=\mathbb{C}$ or $\Delta$, such that $\mathcal{F}=(d z)^{3}$ and $\widetilde{\mathcal{F}}=(d w)^{3}$. Then $\Phi:=w^{-1} \circ z: M \rightarrow M$ is a biholomorphic map such that $f$ and $\widetilde{f} \circ \Phi$ have the same Fubini cubic differential. This yields the required result. 


\subsection{Complex curves and Lagrangian immersions}

On $\mathbb{R}^{4}$, consider the complex structure defined by the identification of $\mathbb{R}^{4}$ with $\mathbb{C}^{2}$ given by

$$
I:{ }^{t}\left(x^{1}, x^{2}, x^{3}, x^{4}\right) \in \mathbb{R}^{4} \mapsto{ }^{t}\left(x^{1}-i x^{2}, x^{3}+i x^{4}\right) \in \mathbb{C}^{2} .
$$

Then, any complex line generates a Lagrangian plane, which implies that a complex immersion $f: M \rightarrow \mathbb{R}^{4} \simeq \mathbb{C}^{2}$ is automatically Lagrangian.

Let $\operatorname{ISL}(2, \mathbb{C}) \cong \mathbb{C}^{2} \rtimes \mathrm{SL}(2, \mathbb{C})$ be the inhomogeneous group associated with the unimodular complex group $\operatorname{SL}(2, \mathbb{C})$. The group $\operatorname{ISL}(2, \mathbb{C})$ can be realized as a closed subgroup of $\mathcal{S}(4, \mathbb{R})$ by

$$
\left(\begin{array}{ll}
1 & 0 \\
v & A
\end{array}\right) \in \operatorname{ISL}(2, \mathbb{C}) \mapsto\left(\begin{array}{cc}
1 & 0 \\
I^{-1} v & {\left[I^{-1} A I\right]}
\end{array}\right) \in \mathcal{S}(4, \mathbb{R}),
$$

where, if we write $A \in \mathrm{SL}(2, \mathbb{C})$ in the form $A=\left(v_{j}^{i}\right)+i\left(w_{j}^{i}\right)$, for $v_{j}^{i}, w_{j}^{i} \in \mathbb{R}$,

$$
\left[I^{-1} A I\right]=\left(\begin{array}{cccc}
v_{1}^{1} & w_{1}^{1} & v_{2}^{1} & -w_{2}^{1} \\
-w_{1}^{1} & v_{1}^{1} & -w_{2}^{1} & -v_{2}^{1} \\
v_{1}^{2} & w_{1}^{2} & v_{2}^{2} & -w_{2}^{2} \\
w_{1}^{2} & -v_{1}^{2} & w_{2}^{2} & v_{2}^{2}
\end{array}\right) .
$$

The Lie algebra $\mathfrak{i s l}(2, \mathbb{C})$ identifies with the Lie subalgebra of $\mathfrak{s}(4, \mathbb{R})$ consisting of all $\mathrm{S}(p, \mathbf{x}) \in$ $\mathfrak{s}(4, \mathbb{R})$ such that

$$
\mathbf{x}=\left(\begin{array}{cc}
a & b \\
c & -{ }^{t} a
\end{array}\right)
$$

where

$$
b, c \in \mathrm{S}(2, \mathbb{R}), \quad \operatorname{tr}(b)=\operatorname{tr}(c)=0, \quad a_{1}^{1}-a_{2}^{2}=0, \quad a_{1}^{2}+a_{2}^{1}=0 .
$$

The precise relation between complex curves and elliptic Lagrangian immersions is summarized in the following statement.

Proposition 4. If $f: M \rightarrow \mathbb{C}^{2}$ is a complex curve without flex points ${ }^{5}$, then $f$ is a totally umbilic elliptic Lagrangian immersion. Conversely, let $f: M \rightarrow \mathbb{R}^{4}$ be a totally umbilic elliptic Lagrangian immersion. Then there exists an affine symplectic transformation $\mathcal{D} \in \mathcal{S}(4, \mathbb{R})$ such that $\mathcal{D} \cdot f$ is a complex curve without flex points.

Proof. Let $f$ be a complex curve without flex points. We already observed that $f$ is Lagrangian. Moreover, since $f$ has no flex points, it is also elliptic. To prove that $f$ is totally umbilic, we have just to observe that the Maurer-Cartan form of an adapted frame along $f$ with respect to a given complex parameter takes values in $i s l(2, \mathbb{C})$. The defining conditions $(7)$ for an adapted frame, combined with (15), yield that the invariant $h$ vanishes identically, and hence $f$ is totally umbilic.

Conversely, let $f$ be a totally umbilic elliptic Lagrangian immersion. For a fixed complex parameter $z: M \rightarrow \mathbb{C}$, let $\mathbf{S}: M \rightarrow \mathcal{S}(4, \mathbb{R})$ be an adapted frame field along $f$. Since $f$ is totally umbilic, using (8), (9) and (10), we find that

$$
\tau^{1}=t_{1} d x-t_{2} d y, \quad \tau^{2}=-t_{2} d x-t_{1} d y
$$

and

$$
\gamma=\left(\begin{array}{cc}
d x & d y \\
d y & -d x
\end{array}\right), \quad \alpha=0, \quad \beta=\left(\begin{array}{cc}
p_{1} d x-p_{2} d y & -p_{2} d x-p_{1} d y \\
-p_{2} d x-p_{1} d y & -p_{1} d x+p_{2} d y
\end{array}\right) .
$$

\footnotetext{
${ }^{5}$ A point $q \in M$ is a flex point if $\left.\left.f_{z}\right|_{q} \wedge f_{z z}\right|_{q}=0, z$ complex coordinate on $M$.
} 
This implies that $\mathbf{S}^{-1} d \mathbf{S}$ is an $\mathfrak{i s l}(2, \mathbb{C})$-valued 1-form of bidegree $(1,0)$, which implies that there exists an element $\mathcal{D} \in \mathcal{S}(4, \mathbb{R})$ such that $\mathcal{D} \mathbf{S}$ is holomorphic and takes values in $\operatorname{ISL}(2, \mathbb{C})$. Therefore, $\mathcal{D} f$ is a complex curve without flex points.

Remark 9. Let $f: M \rightarrow \mathbb{C}^{2}$ be a complex curve. Possibly replacing $f$ with $\mathcal{D} f$, for some $\mathcal{D} \in \mathcal{S}(4, \mathbb{R})$, the homogeneous part $\mathbf{X}: M \rightarrow \mathrm{SL}(2, \mathbb{C})$ of the frame field adapted to a complex coordinate $z$ satisfies

$$
\mathbf{X}^{-1} d \mathbf{X}=\left(\begin{array}{cc}
0 & -i p \\
1 & 0
\end{array}\right) d z
$$

In particular, $f$ can be obtained by integrating the first column vector of $\mathbf{X}$, i.e.

$$
f=\int \mathrm{X}_{1} d z
$$

Note that $\mathbf{X}: M \rightarrow \mathrm{SL}(2, \mathbb{C})$ is a contact complex curve in $\mathrm{SL}(2, \mathbb{C})$. This shows that totally umbilic Lagrangian surfaces are strictly related to the geometry of flat fronts in hyperbolic 3 -space $[13,20]$. For a fixed choice of the complex parameter, let $\mathbf{X}_{\lambda}: M \rightarrow \mathrm{SL}(2, \mathbb{C})$ be so that

$$
\mathbf{X}_{\lambda}^{-1} d \mathbf{X}_{\lambda}=\left(\begin{array}{cc}
0 & -i(p-\lambda) \\
1 & 0
\end{array}\right) d z .
$$

Then, the 1-parameter family of complex curves $f_{\lambda}$ obtained by integrating the first column vector of $\mathbf{X}_{\lambda}$ are not congruent to each other and are all applicable over $f$.

\section{Examples}

The simplest non-umbilic solutions of (14) are given by taking $h$ and $p$ real constants. Possibly rescaling the complex parameter $z$ by a real constant, we may assume $h=1$. The only equation to be solved is then

$$
t_{\bar{z}}=\bar{t} .
$$

The homogeneous part of the Maurer-Cartan form of the adapted frame $\mathbf{S}$ is

$$
\theta=\mathcal{A} d x+\mathcal{B} d y,
$$

where $\mathcal{A}, \mathcal{B} \in \mathfrak{s p}(4, \mathbb{R})$ are given by

$$
\mathcal{A}=\left(\begin{array}{cccc}
1 & 0 & p+1 & 0 \\
0 & -1 & 0 & -(p+1) \\
1 & 0 & -1 & 0 \\
0 & -1 & 0 & 1
\end{array}\right), \quad \mathcal{B}=\left(\begin{array}{cccc}
0 & -1 & 0 & 1-p \\
-1 & 0 & 1-p & 0 \\
0 & 1 & 0 & 1 \\
1 & 0 & 1 & 0
\end{array}\right)
$$

Since $\mathcal{A}$ and $\mathcal{B}$ commute, the general solution of $\mathbf{X}^{-1} d \mathbf{X}$ is

$$
\mathbf{X}(x, y)=\mathbf{Y} \operatorname{Exp}(x \mathcal{A}+y \mathcal{B}),
$$

where $\mathbf{Y} \in \operatorname{Sp}(4, \mathbb{R})$. Without loss of generality, we may assume $\mathbf{Y}=I_{4}$. Then the first two column vectors of $\mathbf{X}$ are

$$
\mathrm{X}_{1}=\left(\begin{array}{c}
\cosh (\sqrt{2-p} y)\left(\cosh (\sqrt{2+p} x)+\frac{\sinh (\sqrt{2+p} x)}{\sqrt{2+p}}\right) \\
-\frac{(\sqrt{p+2} \cosh (\sqrt{p+2} x)+p \sinh (\sqrt{p+2} x)) \sinh (\sqrt{2-p} y)}{\sqrt{4-p^{2}}} \\
\frac{\cosh (\sqrt{2-p} y) \sinh (\sqrt{p+2} x)}{\sqrt{p+2}} \\
\frac{(\sqrt{p+2} \cosh (\sqrt{p+2} x)+2 \sinh (\sqrt{p+2} x)) \sinh (\sqrt{2-p} y)}{\sqrt{4-p^{2}}}
\end{array}\right)
$$


and

$$
\mathrm{X}_{2}=\left(\begin{array}{c}
\frac{(-\sqrt{p+2} \cosh (\sqrt{p+2} x)+p \sinh (\sqrt{p+2} x)) \sinh (\sqrt{2-p} y)}{\sqrt{4-p^{2}}} \\
\cosh (\sqrt{2-p} y)\left(\cosh (\sqrt{2+p} x)-\frac{\sinh (\sqrt{2+p} x)}{\sqrt{2+p}}\right) \\
\frac{(\sqrt{p+2} \cosh (\sqrt{p+2} x)-2 \sinh (\sqrt{p+2} x)) \sinh (\sqrt{2-p} y)}{\sqrt{4-p^{2}}} \\
-\frac{\cosh (\sqrt{2-p} y) \sinh (\sqrt{p+2} x)}{\sqrt{p+2}}
\end{array}\right) .
$$

If $t=t_{1}+i t_{2}$ is a solution of $t_{\bar{z}}=\bar{t}$, that is

$$
\left(t_{1}\right)_{x}-\left(t_{2}\right)_{y}=t_{1}, \quad\left(t_{1}\right)_{y}+\left(t_{1}\right)_{x}=-t_{2},
$$

then the $\mathbb{R}^{4}$-valued 1-form

$$
\mathrm{Y}_{1} d x+\mathrm{Y}_{2} d y=\left(t_{1} \mathrm{X}_{1}-t_{2} \mathrm{X}_{2}\right) d x-\left(t_{2} \mathrm{X}_{1}+t_{1} \mathrm{X}_{2}\right) d y
$$

is closed and the corresponding Lagrangian immersion $f$ can be computed by solving

$$
d f=\mathrm{Y}_{1} d x+\mathrm{Y}_{2} d y=\left(t_{1} \mathrm{X}_{1}-t_{2} \mathrm{X}_{2}\right) d x-\left(t_{2} \mathrm{X}_{1}+t_{1} \mathrm{X}_{2}\right) d y .
$$

This is an elliptic Lagrangian immersion with Fubini's cubic form $\mathcal{F}=t^{2} d z^{3}$. For a fixed $t$ and $p \neq 2$, we have the 1-parameter family of applicable immersions. Explicit solutions of $t_{\bar{z}}=\bar{t}$ can be obtained with the ansatz

$$
t_{1}(x, y)=v_{1}(x)+w_{1}(y), \quad t_{2}(x, y)=v_{2}(x)+w_{2}(y) .
$$

We then have

$$
\begin{array}{ll}
v_{1}(x)=c_{1} e^{2 x}-a_{1}, & v_{2}(x)=c_{2} e^{-2 x}-a_{2}, \\
w_{1}(y)=m_{1} e^{2 y}+m_{2} e^{-2 y}-a_{1}, & w_{2}(y)=-m_{1} e^{2 y}+m_{2} e^{-2 y}+a_{2},
\end{array}
$$

where $a_{1}, a_{2}, c_{1}, c_{2}, m_{1}$ and $m_{2}$ are real constants. In this case, the integration involves elementary functions and can be performed explicitly. The general formulae are rather involved expressions and can be obtained with any standard software of scientific computation such as Mathematica 6.

For instance, if $a_{1}=a_{2}=0$ and $m_{1}=m_{2}=0$, the components of the immersions $f$ are given by

$$
f_{j}(x, y)=\frac{e^{-2 x}}{(p-2) \sqrt{(p+2)\left(4-p^{2}\right)}}\left(\cosh (\sqrt{p+2} x) A_{j}(x, y)+\sinh (\sqrt{p+2} x) B_{j}(x, y)\right),
$$

$j=1, \ldots, 4$, where the functions $A_{j}(y)$ and $B_{j}(y)$ are given by

$$
\begin{aligned}
& A_{1}(x, y)=-c_{1} e^{4 x} \sqrt{(p+2)\left(4-p^{2}\right)} \cosh (\sqrt{2-p} y)-c_{2}\left(p^{2}-4\right) \sinh (\sqrt{2-p} y), \\
& \left.A_{2}(x, y)=-c_{1} e^{4 x}\left(p^{2}-4\right) \sinh (\sqrt{2-p} y)-c_{2} \sqrt{(p+2)\left(4-p^{2}\right)} \cosh (\sqrt{2-p} y)\right), \\
& A_{3}(x, y)=c_{1} e^{4 x} \sqrt{(p+2)\left(4-p^{2}\right)} \cosh (\sqrt{2-p} y) \\
& A_{4}(x, y)=c_{2} \sqrt{(p+2)\left(4-p^{2}\right)} \cosh (\sqrt{2-p} y)
\end{aligned}
$$

and by

$$
\begin{aligned}
& B_{1}(x, y)=c_{1} e^{4 x} p \sqrt{4-p^{2}} \cosh (\sqrt{2-p} y)-c_{2}(p-2) \sqrt{p+2} \sinh (\sqrt{2-p} y), \\
& B_{2}(x, y)=c_{1} e^{4 x}(p-2) \sqrt{p+2} \sinh (\sqrt{2-p} y)-c_{2} p \sqrt{4-p^{2}} \cosh (\sqrt{2-p} y), \\
& B_{3}(x, y)=-2 c_{1} e^{4 x} \sqrt{4-p^{2}} \cosh (\sqrt{2-p} y)-c_{2}(p-2) \sqrt{p+2} \sinh (\sqrt{2-p} y), \\
& B_{4}(x, y)=c_{1} e^{4 x}(p-2) \sqrt{p+2} \sinh (\sqrt{2-p} y)+2 c_{2} \sqrt{4-p^{2}} \cosh (\sqrt{2-p} y) .
\end{aligned}
$$

These provide explicit examples of elliptic Lagrangian immersions with the same Gauss map but which are not applicable and of applicable non-umbilic elliptic Lagrangian immersions. Note that for $p>2$ some of the hyperbolic functions transform into trigonometric functions. 


\section{Acknowledgements}

The work was partially supported by MIUR projects: Metriche riemanniane e varietà differenziabili (E.M.); Proprietà geometriche delle varietà reali e complesse (L.N.); and by the GNSAGA of INDAM. The authors would like to thank the referees for their useful comments and suggestions.

\section{References}

[1] Álvarez Paiva J.C., Durán C.E., Geometric invariants of fanning curves, math.SG/0502481.

[2] Barbot T., Charette V., Drumm T., Goldman W.M., Melnik K., A primer on the (2+1) Einstein universe, arXiv:0706.3055.

[3] Bianchi L., Complementi alle ricerche sulle superficie isoterme, Ann. Mat. Pura Appl. 12 (1905), 20-54.

[4] Blaschke W., Vorlesungen über Differentialgeometrie und geometrische Grundlagen von Einsteins Relativitätstheorie, Bd. 3, bearbeitet von G. Thomsen, J. Springer, Berlin, 1929.

[5] Bryant R.L., A duality theorem for Willmore surfaces, J. Differential Geom. 20 (1984), 23-53.

[6] Burstall F., Hertrich-Jeromin U., Pedit F., Pinkall U., Curved flats and isothermic surfaces, Math. Z. 225 (1997), 199-209, dg-ga/9411010.

[7] Calapso P., Sulle superficie a linee di curvatura isoterme, Rend. Circ. Mat. Palermo 17 (1903), $273-286$.

[8] Cartan É., Sur le problème général de la déformation, C. R. Congrés Strasbourg (1920), 397-406 (or Oeuvres Complètes, III 1, 539-548).

[9] Cartan É., Sur la déformation projective des surfaces, Ann. Scient. Éc. Norm. Sup. (3) 37 (1920), 259-356 (or Oeuvres Complètes, III 1, 441-538).

[10] Chern S.-S., Wang H.-C., Differential geometry in symplectic space. I, Sci. Rep. Nat. Tsing Hua Univ. 4 (1947), 453-477.

[11] Deconchy V., Hypersurfaces in symplectic affine geometry, Differential Geom. Appl. 17 (2002), 1-13.

[12] Fubini G., Applicabilità proiettiva di due superficie, Rend. Circ. Mat. Palermo 41 (1916), 135-162.

[13] Gálvez J.A., Martínez A., Milán F., Flat surfaces in the hyperbolic 3-space, Math. Ann. 316 (2000), 419-435.

[14] Griffiths P.A., On Cartan's method of Lie groups and moving frames as applied to uniqueness and existence questions in differential geometry, Duke Math. J. 41 (1974), 775-814.

[15] Guillemin V., Sternberg S., Variations on a theme by Kepler, American Mathematical Society Colloquium Publications, Vol. 42, American Mathematical Society, Providence, RI, 1990.

[16] Hertrich-Jeromin U., Musso E., Nicolodi L., Möbius geometry of surfaces of constant mean curvature 1 in hyperbolic space, Ann. Global Anal. Geom. 19 (2001), 185-205, math.DG/9810157.

[17] Jensen G.R., Deformation of submanifolds of homogeneous spaces, J. Differential Geom. 16 (1981), 213-246.

[18] Jensen G.R., Musso E., Rigidity of hypersurfaces in complex projective space, Ann. Sci. École Norm. Sup. (4) 27 (1994), 227-248.

[19] Kamran N., Olver P., Tenenblat K., Local symplectic invariants for curves, Commun. Contemp. Math., to appear.

[20] Kokubu M., Umehara M., Yamada K., Flat fronts in hyperbolic 3-space, Pacific J. Math. 216 (2004), 149-175, math.DG/0301224.

[21] McKay B., Lagrangian submanifolds in affine symplectic geometry, Differential Geom. Appl. 24 (2006), 670-689, math.DG/0508118.

[22] Musso E., Deformazione di superfici nello spazio di Möbius, Rend. Istit. Mat. Univ. Trieste 27 (1995), $25-45$.

[23] Musso E., Nicolodi L., Deformation and applicability of surfaces in Lie sphere geometry, Tohoku Math. J. 58 (2006), 161-187, math.DG/0408009.

[24] Musso E., Nicolodi L., Conformal deformation of spacelike surfaces in Minkowski space, Houston J. Math., to appear, arXiv:0712.0807. 